\title{
LIBERAÇÃO DE RESÍDUOS NÃO-EXTRAÍVEIS OU LIGADOS DO HERBICIDA ATRAZINA EM SOLO E SUA ABSORÇÃO POR PLANTAS ${ }^{1}$
}

\author{
LIA EMI NAKAGAWA² e MARA MERCEDES DE ANDRÉA ${ }^{3}$
}

\begin{abstract}
RESUMO - Entre os resíduos de pesticidas em solo podem formar-se resíduos não-extraíveis ou ligados, que podem resultar em menor biodisponibilidade e menor lixiviação do pesticida. Do ponto de vista ambiental, necessita-se saber se estes resíduos podem ser bioliberados e representar contaminação a longo prazo. Investigou-se a liberação de resíduos ligados de ${ }^{14} \mathrm{C}$-atrazina em solo pela adição de inóculo de microrganismos, e a possibilidade de sua absorção por plantas, por meio de técnicas radiométricas. Praticamente não se observou produção de ${ }^{14} \mathrm{CO}_{2}$ (somente de $0 \%$ a $0,8 \%$ ), e a liberação adicional como resíduo extraível a partir de resíduos ligados de ${ }^{14} \mathrm{C}$-atrazina foi pequena (máximo de $3,5 \%$ ). Os valores bioliberados foram semelhantes em amostras de solo esterilizado ou com inóculo de microrganismos. Também não se observou absorção de resíduos ligados por plantas de arroz ou de feijão.
\end{abstract}

Termos para indexação: ação dos pesticidas, microorganismos, mineralização.

\section{RELEASE OF NON-EXTRACTABLE OR BOUND RESIDUES OF THE ATRAZINE HERBICIDE IN SOIL AND ITS ABSORPTION BY PLANTS}

\begin{abstract}
Among the pesticide residues in soil, the non-extractable or bound residues may be formed resulting in less bioavailability and less leaching of the pesticide residues. From the environmental point of view the knowledge on the possibility of the biorelease of these residues is needed for evaluations on long term pollution. The release of ${ }^{14} \mathrm{C}$-atrazine bound residues was investigated in soil by addition of inocula with microorganisms and the possibility of their absorption by plants through radiometric techniques. The ${ }^{14} \mathrm{CO}_{2}$ production almost did not happen (only from $0 \%$ to $0.8 \%$ ) and additional release of extractable residue from bound residues of ${ }^{14} \mathrm{C}$-atrazine was small (until $3.5 \%$ ). The bioreleased values were near the same in sterilized or with inocula of microorganisms soil samples. The uptake of bound residues was not observed neither by rice or bean plants.
\end{abstract}

Index terms: pesticide actions, microorganisms, mineralization.

\section{INTRODUÇÃO}

A avaliação dos possíveis prejuízos ambientais causados pela presença de um pesticida no solo depende de estudos em que se estabeleça o destino final deste pesticida. Entre os pesticidas, a atrazina tem sido o herbicida mais usado durante os últimos 30 anos no controle não-seletivo de ervas daninhas

\footnotetext{
${ }^{1}$ Aceito para publicação em 27 de março de 2000.

Extraído da dissertação de mestrado do primeiro autor, apresentada ao Instituto de Biociências (IB), Universidade de São Paulo (USP).

${ }^{2}$ Biól., M.Sc., Centro de Proteção Ambiental, IB, Caixa Postal 12.898, CEP 04010-970 São Paulo. E-mail: nakagawa@biologico.br

${ }^{3}$ Biól., Dra., Centro de Proteção Ambiental, IB. E-mail: andrea@biologico.br
}

em solo não cultivado e para controle seletivo de ervas daninhas em culturas, como: milho, sorgo, canade-açúcar e abacaxi (Radosevich et al., 1995). Assim, o estudo do comportamento da atrazina no solo é de interesse prático, dadas as possibilidades de contaminação ambiental, e também para possibilitar o melhor uso (Graham-Bryce, 1981).

O desaparecimento aparente de um pesticida do solo pode ocorrer devido à incapacidade de se detectarem resíduos por procedimentos convencionais, como a extração (Lichtenstein, 1980); mas a utilização de técnicas nucleares utilizando pesticidas radiomarcados possibilitou a detecção de resíduos ligados ou não-extraíveis, que não eram aferidos por nenhum método analítico (Klein \& Scheunert, 1982).

O resíduo ligado é formado pelas ligações químicas ou físicas entre a molécula do pesticida e a matriz 
do solo, e tem sido detectado em todas as classes de pesticidas, em quantidades que variam de $20 \%$ a $70 \%$ do composto aplicado. Os processos de adsorção e formação de resíduo ligado podem ter como consequiências variações no seu comportamento, como: diminuição da degradação do pesticida (Calderbank, 1989), perda da sua atividade biológica (Kaufman, 1976) e da sua identidade química (Calderbank, 1989), e alteração do escoamento superficial e da lixiviação deste pesticida. Estes resíduos podem, ainda, representar uma reserva potencial de compostos que podem interagir com outros componentes do ecossistema e exercer atividade biológica (Yee et al., 1985).

Vários estudos indicam que, de fato, o resíduo ligado pode ser liberado por microrganismos ou por processos químicos, tempos após sua formação e detecção. Khan \& Ivarson (1981) obtiveram liberação de $27 \%$ de resíduo de prometrina ligado ao solo na forma de resíduo extraível, e produção de $0,01 \%$ de ${ }^{14} \mathrm{CO}_{2}$. Gerstl \& Helling (1986) obtiveram liberação de, aproximadamente, $0,9 \%$ de resíduo ligado de paration metílico na forma extraível e produção de, aproximadamente, $12 \%$ de ${ }^{14} \mathrm{CO}_{2}$. Andréa \& Wiendl (1995) obtiveram liberação de 1,7\% a 9,8\% dos resíduos ligados de paration etílico na forma extraível, e de 0 a $1 \%$ de produção de ${ }^{14} \mathrm{CO}_{2}$, dependendo do tipo de solo.

Vários estudos também detectaram a absorção destes resíduos por plantas (Khan \& Marriage, 1977; Fuhremann \& Lichtenstein, 1978; Magallona et al., 1986; Murthy \& Raghu, 1988; Bertin et al., 1990; Khan, 1991; Verma \& Pillai, 1991; Andréa \& Wiendl, 1995 ), mas a absorção está, de modo geral, abaixo de $1 \%$ da quantidade de resíduo inicialmente ligado no solo (Klein \& Scheunert, 1982). A absorção de resíduos ligados de pesticidas por plantas pode ter como consequiência sua transferência para culturas posteriores e transferência nas cadeias alimentares.

Estudou-se, neste trabalho, a possibilidade de bioliberação de resíduos ligados de atrazina em solo através da ação da microbiota, bem como a possibilidade de sua absorção por plantas.

\section{MATERIAL E MÉTODOS}

A liberação de resíduos ligados de atrazina por microrganismos foi estudada em três tipos de solo contendo re- síduos ligados de atrazina e com adição de três tipos de inóculo com microrganismos.

Utilizaram-se amostras de dois solos Glei Húmico (GH), um de Campinas, SP, e outro, de São Paulo, SP. O solo de Campinas é proveniente de experimento anterior conduzido em campo (Nakagawa et al.,1996), e o de São Paulo é proveniente de experimento de dissipação de atrazina, conduzido em laboratório (Nakagawa \& Andréa, 2000). Utilizou-se, também, amostra de um Latossolo Vermelho Escuro (LE), também de Campinas. Todas as amostras continham ${ }^{14} \mathrm{C}$-resíduos previamente ligados do herbicida atrazina, com quantidades de radioatividade de $0,059 \mathrm{kBq} \mathrm{g}^{-1}, 0,067 \mathrm{kBq} \mathrm{g}^{-1}$ e $0,059 \mathrm{kBq} \mathrm{g}^{-1}$, respectivamente. As características físico-químicas dos solos são: solo GH de Campinas: pH 4,6, $36 \mathrm{~g} \mathrm{dm}^{-3}$ de matéria orgânica, $279 \mathrm{~g} \mathrm{~kg}^{-1}$ de argila, $630 \mathrm{~g} \mathrm{~kg}^{-1}$ de areia; solo GH de São Paulo: $\mathrm{pH} 4,8,36 \mathrm{~g} \mathrm{dm}^{-3}$ de matéria orgânica, $660 \mathrm{~g} \mathrm{~kg}^{-1}$ de argila, $280 \mathrm{~g} \mathrm{~kg}^{-1}$ de areia; e solo LE: $\mathrm{pH} 5,4,36 \mathrm{~g} \mathrm{dm}^{-3}$ de matéria orgânica, $449 \mathrm{~g} \mathrm{~kg}^{-1}$ de argila, e 40,2 $\mathrm{g} \mathrm{kg}^{-1}$ de areia.

Amostras destes solos foram esterilizadas por exposição à radiação gama (dose total de 4,5 Mrad e taxa de dose de 1,5 $\mathrm{Mrad} \mathrm{h}^{-1}$, pela Empresa Brasileira de Radiação, que mostrou ser o método mais apropriado para esterilização de amostra contendo resíduos ligados (Nakagawa \& Andréa, 1997).

A possibilidade de liberação de resíduos ligados presentes foi verificada por meio de metabolismo microbiano utilizando-se os seguintes inóculos: 1- suspensão de solo GH de São Paulo incubado por 56 dias apenas com água; 2- suspensão de solo GH de São Paulo incubado por 56 dias com atrazina; 3- suspensão de solo GH de São Paulo incubado por 56 dias com atrazina e contendo apenas Pseudomonas putida.

Antes do início do experimento, mediu-se a atividade microbiana dos inóculos por meio de radiorrespirometria de ${ }^{14} \mathrm{C}$-glicose e contagem do número de unidades formadoras de colônias - UFC, conforme Nakagawa \& Andréa (2000).

Às três amostras (30 g) de cada tipo de solo adicionouse inóculo na forma de suspensão aquosa de solo fresco, cujo volume correspondeu a $50 \%$ da capacidade máxima de retenção de água (CMRA). Outras três amostras de cada solo foram reativadas apenas com água (50\% CMRA). Pequenos recipientes contendo $10 \mathrm{~mL}$ de solução de $\mathrm{KOH}$ 0,2 M foram colocados no interior dos frascos com solo, para captação de ${ }^{14} \mathrm{CO}_{2}$ liberado a partir dos resíduos de ${ }^{14} \mathrm{C}$-atrazina ligados aos solos. 
Todas as amostras foram incubadas por 56 dias, no escuro, em câmara de temperatura controlada a, aproximadamente, $29^{\circ} \mathrm{C}$. Durante este período, a solução de $\mathrm{KOH}$ foi coletada e analisada semanalmente, e substituída por uma solução recém-preparada.

No início e ao final do período de estudo, o próprio solo foi analisado quanto às quantidades de radiocarbono presente, reextraível e que permaneceu ligado. Para isto, subamostras de $500 \mathrm{mg}$ de solo foram submetidas a combustão conforme Nakagawa \& Andréa (2000), para determinação do radiocarbono presente nas amostras de solo. O solo restante das amostras foi extraído por oito horas em soxhlet com $150 \mathrm{~mL}$ de metanol:água $(8: 2 \mathrm{v} / \mathrm{v})$, para detecção do radiocarbono extraível. A diferença entre o radiocarbono extraível no início do experimento (T.0) e o radiocarbono extraível após o período de incubação (T.56) foi considerada como decorrente da liberação de radiocarbono ligado na forma de resíduo novamente extraível. Subamostras dos solos extraídos também foram submetidas a combustão, para determinação dos resíduos ligados (não-extraíveis) ainda presentes no solo. A radioatividade de todas as amostras foi quantificada por contagem de cintilação em líquido (LSC), conforme Nakagawa e Andréa (2000).

Três repetições de amostras de solo GH de Campinas e de solo LE contendo resíduos ligados de atrazina foram reumedecidas por subirrigação e distribuídas em vasos de plástico em que foram plantadas três plântulas de feijão (Phaseolus vulgaris $\mathrm{L}$.) ou duas de arroz (Oryza sativa L.). Foram utilizadas $400 \mathrm{~g}$ de solo para o experimento com feijão, e $20 \mathrm{~g}$ para o experimento com arroz. Outro grupo de vasos recebeu somente solo contendo os resíduos ligados. Os vasos foram mantidos em laboratório à temperatura e luminosidade ambiente, por 14 dias. A seguir, as plantas foram coletadas e pesadas, e amostras de $100 \mathrm{mg}$ de tecido vegetal foram submetidas a combustão, para determinação do radiocarbono total presente na planta. Amostras de $500 \mathrm{mg}$ de solo dos vasos foram também submetidas a combustão, para determinação da quantidade de radiocarbono ainda presente no solo.

Todos os resultados foram analisados estatisticamente, por meio de cálculo de médias e desvio-padrão.

\section{RESULTADOS E DISCUSSÃO}

A atividade microbiana dos inóculos resultou em $5,3 \%$ de produção de ${ }^{14} \mathrm{CO}_{2}$ a partir de ${ }^{14} \mathrm{C}$-glicose no inóculo de suspensão de solo (inóculo 1), 5,1\% no inóculo de suspensão de solo incubado com atrazina (inóculo 2), e 15,2\% no inóculo de suspen- são de solo incubado com atrazina e $P$. putida (inóculo 3). Paralelamente, o número de colônias bacterianas (UFC) presente nos inóculos a serem adicionados aos solos foi de $2,3 \times 10^{5}$ UFC no inóculo 1; 2,6 x $10^{5} \mathrm{UFC}$, no inóculo 2; e 6,0 × $10^{5} \mathrm{UFC}$, no inóculo 3. Portanto, todos os inóculos mostraram atividade e presença de UFC, e podem ser usados como agentes microbianos para o processo de liberação de resíduos ligados.

Após o período de incubação de 56 dias, praticamente não houve desprendimento de radiocarbono como ${ }^{14} \mathrm{CO}_{2}$ a partir de resíduos ligados de ${ }^{14} \mathrm{C}$-atrazina nos solos estudados, e houve um máximo de $0,8 \%$ de ${ }^{14} \mathrm{CO}_{2}$ produzido (Tabela 1). Além disso, em nenhum dos casos houve diferença entre solo esterilizado e solo com inoculação de solo ou de solo previamente exposto à atrazina.

Comparando-se os resultados obtidos no início do experimento (T.0) com os de 56 dias, isto é, após o período de incubação, verificou-se que no solo GH de Campinas e no solo LE não houve liberação na forma de radiocarbono extraível. No solo GH de São Paulo, por outro lado, houve pequena liberação como radiocarbono extraível (máximo de 3,5\%). Entretanto, esta liberação não foi maior após a adição de inóculos (Tabela 1).

Portanto, independentemente do tipo de solo, da adição, ou não, de inóculo ao solo, e do tipo de inóculo adicionado, observou-se que praticamente não houve produção de ${ }^{14} \mathrm{CO}_{2}$, e a liberação de radiocarbono ligado como resíduo novamente extraível foi pequena, sendo que a grande maioria dos resíduos detectados permaneceu como resíduos ainda ligados (Tabela 1).

Embora outros estudos indiquem que os microrganismos podem ser os causadores da liberação de resíduos ligados de outros pesticidas (Khan \& Ivarson, 1981, 1982; Racke \& Lichtenstein, 1985; Khan \& Behki, 1990; Musumeci \& Ostiz, 1994; Andréa $\&$ Wiendl, 1995 ), no herbicida atrazina, nas condições deste experimento, os microrganismos não tiveram papel considerável na liberação de resíduos ligados. Assim, mesmo microrganismos comprovadamente capazes de degradar a atrazina não foram capazes de liberar resíduos ligados de ${ }^{14} \mathrm{C}$-atrazina do solo. 
TABELA 1. Recuperação total do radiocarbono em solos Glei Húmico (GH) de Campinas, Glei Húmico (GH) de São Paulo e Latossolo Vermelho-Escuro (LE) contendo resíduos ligados de ${ }^{14} \mathrm{C}$-atrazina, após 56 dias de incubação; os dados representam porcentagens médias da atividade presente inicialmente como resíduos ligados \pm desvio-padrão.

\begin{tabular}{|c|c|c|c|c|c|}
\hline \multirow[t]{2}{*}{ Solo } & \multirow[t]{2}{*}{ Tratamento $^{1}$} & \multirow[t]{2}{*}{$\Sigma^{14} \mathrm{CO}_{2}$ no período } & \multicolumn{2}{|c|}{${ }^{14} \mathrm{C}$ - extraível } & \multirow[t]{2}{*}{${ }^{14} \mathrm{C}$ - ainda ligado } \\
\hline & & & T.0 & T.56 & \\
\hline \multirow[t]{4}{*}{ GH de Campinas } & Esterilizado & $0,0 \pm 0,0$ & $6,9 \pm 2,0$ & $6,9 \pm 1,9$ & $95,8 \pm 4,3$ \\
\hline & 1 & $0,2 \pm 0,4$ & & $7,2 \pm 1,9$ & $87,6 \pm 5,0$ \\
\hline & 2 & $0,2 \pm 0,3$ & & $7,2 \pm 1,2$ & $89,5 \pm 3,4$ \\
\hline & 3 & $0,0 \pm 0,0$ & & $6,7 \pm 4,1$ & $93,7 \pm 4,1$ \\
\hline \multirow[t]{4}{*}{ GH de São Paulo } & Esterilizado & $0,0 \pm 0,0$ & $11,1 \pm 1,6$ & $12,6 \pm 2,7$ & $93,0 \pm 5,2$ \\
\hline & 1 & $0,6 \pm 0,2$ & & $14,6 \pm 2,1$ & $90,2 \pm 3,2$ \\
\hline & 2 & $0,8 \pm 0,3$ & & $12,4 \pm 0,9$ & $94,5 \pm 2,7$ \\
\hline & 3 & $0,4 \pm 0,3$ & & $8,6 \pm 2,3$ & $100,3 \pm 6,7$ \\
\hline \multirow[t]{4}{*}{ LE } & Esterilizado & $0,0 \pm 0,0$ & $8,3 \pm 2,2$ & $6,4 \pm 2,3$ & $88,3 \pm 6,7$ \\
\hline & 1 & $0,4 \pm 0,7$ & & $7,2 \pm 2,0$ & $98,5 \pm 5,1$ \\
\hline & 2 & $0,4 \pm 0,6$ & & $5,4 \pm 0,6$ & $96,5 \pm 3,2$ \\
\hline & 3 & $0,0 \pm 0,0$ & & $4,1 \pm 1,6$ & $98,3 \pm 6,8$ \\
\hline
\end{tabular}

1 1: solo com inoculação de solo GH de São Paulo, previamente incubado por 56 dias; 2: solo com inoculação de solo GH de São Paulo, previamente incubado por 56 dias com atrazina; 3: solo com inoculação de Pseudomonas putida, previamente incubado por 56 dias com atrazina.

Após 14 dias do plantio de plântulas de feijão ou arroz nas amostras de solos com resíduos ligados de atrazina, detectou-se radiocarbono apenas nos solos. Portanto, não houve absorção de radiocarbono a partir de resíduos ligados, presentes nos solos, pelas plantas de arroz ou de feijão, e os resíduos previamente ligados permaneceram nos solos. Dados de literatura (Klein \& Scheunert, 1982), também dão conta de baixos valores de absorção de resíduos ligados de vários pesticidas por plantas, embora a absorção de resíduos ligados de atrazina não tenha sido anteriormente estudada.

\section{CONCLUSÕES}

1. A produção de ${ }^{14} \mathrm{CO}_{2}$ e a liberação de resíduos ligados na forma de resíduos extraíveis são muito pequenas e semelhantes nos três tipos de amostras de solos estudados, e não há influência de microrganismos na liberação destes resíduos ligados.
2. Plantas de feijão ou de arroz não liberam nem absorvem resíduos ligados de atrazina do solo.

3. O risco de contaminação ambiental a partir de resíduos ligados de atrazina a solos Glei Húmico e Latossolo Vermelho-Escuro é mínimo.

\section{AGRADECIMENTOS}

À Empresa Brasileira de Radiação pela irradiação das amostras de solo; à CAPES, pela concessão da bolsa de estudos ao primeiro autor; à Dra. Vivian Pellizari, do Laboratório de Microbiologia Ambiental do ICB/USP, pela reativação das culturas de Pseudomonas putida.

\section{REFERÊNCIAS}

ANDRÉA, M.M.; WIENDL, F.M. Formation and biorelease of bound residues of pesticides in two Brazilian soils. II. $\left[{ }^{14} \mathrm{C}\right]$-parathion. Pesquisa Agropecuária Brasileira, Brasília, v.30, n.5, p.695700, maio 1995. 
BERTIN, G.; SCHIAVON, M.; POTTIER, C. Plant bioavailability of "natural" and "model" humic acidbound $\left[{ }^{14} \mathrm{C}\right]$ atrazine residues. Toxicological and Environmental Chemistry, Chur, v.26, p.203-210, 1990.

CALDERBANK, A. The occurrence and significance of bound pesticide residues in soil. Reviews of Environmental Contamination and Toxicology, New York, v.108, p.71-103, 1989.

FUHREMANN, T.W.; LICHTENSTEIN, E.P. Release of soil-bound methyl $\left[{ }^{14} \mathrm{C}\right]$ parathion residues and their uptake by earthworms and oat plants. Journal of Agricultural and Food Chemistry, Washington, v.26, p.605-610, 1978.

GERSTL, Z.; HELLING, C.S. Comparative extraction techniques for determining bound residues in soil treated with allyl alcohol and hexachlorobenzene. In: INTERNATIONAL ATOMIC ENERGY AGENCY (Vienna, Austria). Quantification, nature and bioavailability of bound ${ }^{14} \mathrm{C}$-pesticide residues in soil, plants and food. Vienna, 1986. p.121-136.

GRAHAM-BRYCE, I.G. The behaviour of pesticides in soil. In: GREENLAND, D.J.; HAYES, M.H.B. (Ed.). The chemistry of soil processes. New York : J. Wiley, 1981. p.621-670.

KAUFMAN, D.D. Bound and conjugated pesticide residues. In: KAUFMAN, D.D.; STILL, G.G.; PAULSON, G.D.; BANDAL, S.K. (Ed.). Bound and conjugated pesticide residues. Washington : American Chemical Society, 1976. p.1-10. (ACS Symposium Series, 29).

KHAN, S.U. Bound (non-extractable) pesticide degradation products in soils: biovailability to plants. In: SOMASUNDARAM, L.; COATS, J.R. (Ed.). Pesticide transformation products: fate and significance in the environment. Washington : American Chemical Society, 1991.p.108-121. (ACS Symposium Series, 459).

KHAN, S.U.; BEHKI, R.M. Effects of Pseudomonas species on the release of bound ${ }^{14} \mathrm{C}$ residues from soil treated with $\left[{ }^{14} \mathrm{C}\right]$ atrazine. Journal of Agricultural and Food Chemistry, Washington, v.38, p.2090-2093, 1990.

KHAN, S.U.; IVARSON, K.C. Microbiological release of unextracted (bound) residues from an organic soil treated with prometryn. Journal of Agricultural and Food Chemistry, Washington, v.29, p.13011303, 1981.

KHAN, S.U.; IVARSON, K.C. Release of soil bound (nonextractable) residues by various physiological groups of microorganisms. Journal of Environmental Science and Health, New York, v.B17, n.6, p.737749, 1982.

KHAN, S.U.; MARRIAGE, P.B. Residues of atrazine and its metabolites in an orchard soil and their uptake by oat plants. Journal of Agricultural and Food Chemistry, Washington, v.25, n.6, p.1408-1413, 1977.

KLEIN, W.; SCHEUNERT, I. Bound pesticide residues in soil, plants and food with particular emphasis on the application of nuclear techniques. In: INTERNATIONAL ATOMIC ENERGY AGENCY (Vienna, Austria). Agrochemicals: fate in food and environment. Vienna, 1982. p.177-205.

LICHTENSTEIN, E.P. Bound residues in soils and transfer of soil residues in crops. Residue Reviews, New York, v.76, p.147-153, 1980.

MAGALLONA, E.D.; BAJET, C.M.; BARREDO, M.J.V. Bound residues of isoprocarb in a rice paddy ecosystem. In: INTERNATIONAL ATOMIC AGENCY (Vienna, Austria). Quantification, nature and bioavailability of bound ${ }^{14} \mathrm{C}$-pesticide residues in soil, plants and food. Vienna, 1986. p.57-63.

MURTHY, N.B.K.; RAGHU, K. Soil bound residues of carbaryl and 1-naphtol: release and mineralization in soil, and uptake by plants. Journal of Environmental Science and Health, New York, v.B23, n.6, p.575-585, 1988.

MUSUMECI, M.R; OSTIZ, S.B. Binding of cypermethrin residue in Brazilian soils and its release by microbial activity. Revista de Microbiologia, São Paulo, v.25, p.216-219, 1994.

NAKAGAWA, L.E.; ANDRÉA, M.M. Degradação e formação de resíduos não-extraíveis ou ligados do herbicida atrazina em solo. Pesquisa Agropecuária Brasileira, Brasília, v.35, n.8, p.1509-1515, ago. 2000.

NAKAGAWA, L.E.; ANDRÉA, M.M. Liberação de resíduos ligados de atrazina de solos por meio de esterilização por autoclavagem e radiação gama. Revista Brasileira de Ciência do Solo, Campinas, v.21, p.707-710, 1997.

Pesq. agropec. bras., Brasília, v.35, n.8, p.1517-1522, ago. 2000 
NAKAGAWA, L.E.; LUCHINI, L.C.; MUSUMECI, M.R.; MATALLO, M.B. Behaviour of atrazine in soils of tropical zone: degradation, mobility and uptake of atrazine residues from soils in a crop rotation system (maize/beans). Journal of Environmental Science and Health, New York, v.B31, n.2, p.203-224, 1996.

RACKE, K.D.; LICHTENSTEIN, E.P. Effects of soil microorganisms on the release of bound ${ }^{14} \mathrm{C}$ residues from soils previously treated with $\left[{ }^{14} \mathrm{C}\right]$ parathion. Journal of Agricultural and Food Chemistry, Washington, v.33, p.938-943, 1985.
RADOSEVICH, M.; TRAINA, S.J.; HAO, Y.L.; TUOVINEN, O.H. Degradation and mineralization of atrazine by a soil bacterial isolate. Applied Environmental Microbiology, Washington, v.61, n.1, p.297-302, 1995.

VERMA, A.; PILLAI, M.K.K. Bioavailability of soilbound residues of DDT and $\mathrm{HCH}$ to certain plants. Soil Biology and Biochemistry, Oxford, v.23, n.4, p.346-351, 1991.

YEE, D.; WEINBERGER, P.; KHAN S.U. Release of soil-bound prometryne residues under different soil $\mathrm{pH}$ and nitrogen fertilizer regimes. Weed Science, Lawrence, v.33, p.882-887, 1985. 